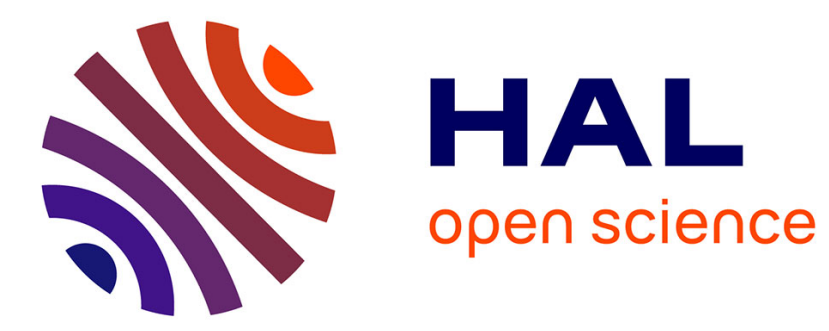

\title{
Anisotropic magnetic molecular dynamics of cobalt nanowires
}

David Beaujouan, Pascal Thibaudeau, Cyrille Barreteau

\section{To cite this version:}

David Beaujouan, Pascal Thibaudeau, Cyrille Barreteau. Anisotropic magnetic molecular dynamics of cobalt nanowires. Physical Review B: Condensed Matter and Materials Physics (1998-2015), 2012, 86, pp.174409. 10.1103/PhysRevB.86.174409 . cea-01477524

\section{HAL Id: cea-01477524 https://hal-cea.archives-ouvertes.fr/cea-01477524}

Submitted on 27 Feb 2017

HAL is a multi-disciplinary open access archive for the deposit and dissemination of scientific research documents, whether they are published or not. The documents may come from teaching and research institutions in France or abroad, or from public or private research centers.
L'archive ouverte pluridisciplinaire HAL, est destinée au dépôt et à la diffusion de documents scientifiques de niveau recherche, publiés ou non, émanant des établissements d'enseignement et de recherche français ou étrangers, des laboratoires publics ou privés. 


\title{
Anisotropic magnetic molecular dynamics of cobalt nanowires
}

\author{
David Beaujouan, ${ }^{1,2}$ Pascal Thibaudeau, ${ }^{1, *}$ and Cyrille Barreteau ${ }^{2}$ \\ ${ }^{1}$ CEA/Le Ripault, DAM, BP16, F-37260 Monts, France \\ ${ }^{2}$ CEA/Saclay, IRAMIS, F-91191 Gif-sur-Yvette, France \\ (Received 19 September 2012; published 12 November 2012)
}

\begin{abstract}
An investigation of thermally induced spin and lattice dynamics of a cobalt nanowire on a (111)Pt substrate is presented via magnetic molecular dynamics. This dynamical simulation model treats each atom as a particle supporting a classical spin. A coordinate dependent on both exchange and anisotropic functions ensures a minimal coupling between the spin and the lattice degrees of freedom to translate the magnetostrictive behavior of most magnetic materials. A spin-pair model of anisotropy is proposed to connect to the lattice thermodynamics. In order to solve linked spin-coordinate equations of motion, the efficiencies of algorithms based on SuzukiTrotter decompositions are compared. The temperature dependence of the magnetic behavior of Co nanowires is investigated through thermal stochastic connections with mechanical and spin Langevin noises. From a magnetic Hamiltonian parametrized on ab initio calculations, the size dependence of the energy barriers and characteristic time scales of the magnetization relaxation are computed. In the superparamagnetic limit, it is shown that all spins in a nanowire evolve in a coherent rotation. When the size of the single nanowire increases, nucleations of domain walls let the activation energy be independent of the length of the wire.
\end{abstract}

DOI: 10.1103/PhysRevB.86.174409

PACS number(s): 78.20.Bh, 75.80.+q, 75.60.Jk, 75.10.Hk

\section{INTRODUCTION}

In many important areas of engineering, there is increasing interest in novel magnetic nanomaterials. ${ }^{1}$ With the neverending miniaturization quest of the electronics industry, ${ }^{2}$ there is a crucial need for a detailed atomistic description of magnetic materials. Understanding the role of magnetic effects in the structural stability of some metals is still a challenge. However, it has been shown recently that some phase transitions of iron, driven by spin fluctuations, have effects on the stability of dislocation loops. ${ }^{3}$ Therefore a true atomistic simulation of a magnetic materials at finite temperature has to conciliate the dynamics of both the spin and the lattice degrees of freedom. ${ }^{4-6}$

From the Bohr-van Leeuwen theorem and without internal degrees of freedom, the magnetization of a classical system is always null. ${ }^{7}$ Because magnetism is a quantum effect, the magnetic molecular dynamics (MMD) cannot mimic the magnetization dynamics without introducing the spins as supplemental dynamical variables. In a classical fluid theory, the first formulation of the dynamics of classical particles with spin degrees of freedom was given early by Turski. ${ }^{8}$ More recently, another coupling of the molecular dynamics (MD) and atomic spin dynamics was presented by Antropov et al. ${ }^{9,10}$ This approach is based simultaneously on a quantum mechanical derivation of localized moment and atomic equations of motion but is a colossal consumption of computational resources. However, in simplifying the full set of first-principles equations of motion, Akbar et al. ${ }^{11}$ have demonstrated a few-hundred-atom simulation in which complex antiferromagnetic order and helical structures are found for $\gamma$-Fe. Moreover, explicit calculations of spin damping have been reported on bulk, monolayers, and atomic wires of Fe, $\mathrm{Co}$, and $\mathrm{Ni}^{12}$

To circumvent the computer demands used by firstprinciples methods, Ackland, ${ }^{13}$ then Dudarev and Derlet, ${ }^{14}$ has proposed to derive magnetic interatomic potentials suitable for atomistic simulations. They use the second-moment functional form of the empirical many-body potential and incorporate an explicit magnetic term in it, which has to be solved self-consistently to describe the magnetic energy gained by a low-coordination-number environment. Even if this method allows a good translation of the mechanical stability of transition metals, it is restricted to collinear spin configurations and does not permit us to follow in time the direction of each magnetic moment.

Thus the whole dynamical behavior of a magnetic solid can be seen as an exchange among three thermodynamical reservoirs (electronic, spin and lattice) as shown in Fig. 1.

Although the ultrafast dynamics of a magnetic material is determined by its spin-dependent electronic structure, the time resolution of all-optical pump-probe experiments prevents the need for detailed electronic dynamics. ${ }^{15}$ In this situation, the electrons are considered at equilibrium during the probe time. This allows us to focus on the coupled spin and lattice dynamics and a way to unify them in agreement with their similar typical time steps. ${ }^{16}$

In restricting between spin and lattice excitations, Omelyan and coworkers have developed high-order integration algorithms for both $\mathrm{MD}^{17-19}$ and spin-lattice dynamics. ${ }^{20}$ Implementation on a classical $X Y$-spin fluid in an external magnetic field was followed. ${ }^{21}$ Ma et al. ${ }^{22,23}$ have implemented these algorithms to develop spin-lattice dynamics simulations of $\gamma$-Fe. In these simulation schemes, the spin and the lattice degrees of freedom are treated at the same level. The dynamics of these coupled classical variables constitutes what we call MMD. In MMD because of the virial theorem, exchanges between spin and lattice reservoirs result from the spatial dependence of exchange interaction and magnetic anisotropy, which lets additional magnetic forces vary the kinetic temperature. Such a spatial dependence of the magnetic anisotropy allows magnetoelasticity to be included ${ }^{24,25}$ within the MMD. Even if the exchange energy considered by Ma et al. ${ }^{26}$ has a spatial dependence, because of its isotropic nature, it is unable to capture any directional magnetostrictive behavior. 


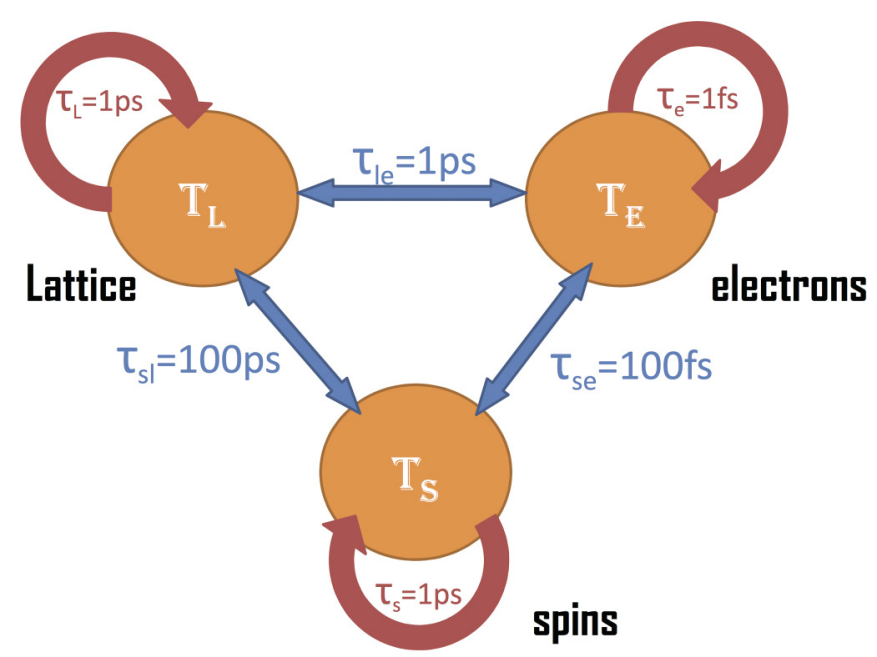

FIG. 1. (Color online) The lattice, electron, and spin thermodynamic reservoirs with their corresponding temperatures $\left(T_{L}, T_{E}\right.$, and $T_{S}$ ). Arrows show the exchange of energy between each reservoir and their typical relaxation times.

Section II describes the total Hamiltonian of the system, combining the mechanical and magnetic parts. Its parametrization is discussed on the basis of $a b$ initio calculations. In Sec. III, several integration algorithms are derived. These algorithms are based on geometric integration with SuzukiTrotter (ST)-like decompositions of the evolution operator of the position, impulsion, and spin variables. Because of the noncommutative character of the time integration, the order in the ST-like decomposition is discussed in order to optimize the numerical speed, stability, and accuracy. By using a Langevin dynamics combined within the fluctuationdissipation theorem, the connection between the kinetic and the magnetic temperature is presented. Finally, Sec. IV illustrates the superparamagnetic behavior of an atomic chain of cobalt through MMD simulations.

\section{MMD HAMILTONIAN}

A model which aspires to reproduce the general behavior of a $3 d$ magnetic transition metal at finite temperature has to include not only the mechanical interactions between atoms but also the magnetic coupling between their neighboring spins. ${ }^{27}$ If the first interaction is rather common within the implementation of an MD using many-body potentials, the second is much more difficult since one needs a description not only of the spin-spin interaction but also of its modification by the local atomic environment. Nevertheless, by introducing the notion of average magnetization into a given volume, ${ }^{28}$ interaction between the spins and the lattice can be partially taken into account with effective approximations.

\section{A. Mechanical and anisotropic exchange Hamiltonian}

To perform our MMD, a classical system composed of $N$ magnetic particles of mass $m$ is considered and described by the following pair Hamiltonian:

$$
\begin{aligned}
\mathcal{H}= & \sum_{i=1}^{N} \frac{\left\|\vec{p}_{i}\right\|^{2}}{2 m_{i}}+\sum_{i<j}^{N} V\left(r_{i j}\right)-\sum_{i<j}^{N} J\left(r_{i j}\right) \vec{s}_{i} \cdot \vec{s}_{j} \\
& -\sum_{i<j}^{N} K\left(r_{i j}\right)\left[\left(\hat{r}_{i j} \cdot \vec{s}_{i}\right)\left(\hat{r}_{i j} \cdot \vec{s}_{j}\right)-\frac{1}{3}\left(\vec{s}_{i} \cdot \vec{s}_{j}\right)\right] \\
& -\sum_{i<j}^{N} \vec{D}\left(r_{i j}\right) \cdot\left(\vec{s}_{i} \times \vec{s}_{j}\right)-g \mu_{B} \mu_{0} \sum_{i=1}^{N} \vec{H}_{\mathrm{ext}} \cdot \vec{s}_{i} .
\end{aligned}
$$

Here $\vec{r}_{i}, \vec{p}_{i}$, and $\vec{s}_{i}$ are, respectively, the position, impulsion, and spin direction of the $i$ th particle. $r_{i j}$ is the distance between between particle $i$ and particle $j$, while $\hat{r}_{i j}$ is the corresponding unit vector. The first term is the kinetic energy and the mechanical potential is denoted by $V\left(r_{i j}\right)$, which is supposed to generate only central forces. In the same way, $J\left(r_{i j}\right)$, $K\left(r_{i j}\right)$, and $D\left(r_{i j}\right)$ are, respectively, the diagonal exchange interaction and the amplitudes of the magnetic anisotropic for both a symmetric pseudodipolar and an antisymmetric (Dzyaloshinskii-Moriya) interaction for a couple of spins at the considered distance $r_{i j}$. A single family of magnetic atoms is thus considered $\vec{s}_{i} \cdot \vec{s}_{i}=1$, which provides a convenient scaling of $g \mu_{B} / \hbar$ to find the corresponding magnetic moment, where $\vec{H}_{\text {ext }}$ is the Zeeman static magnetic field. Previous models have considered only a symmetric isotropic exchange..$^{20,26,29}$ Many types of two-site anisotropy have been proposed in the literature, ${ }^{27}$ but the pseudodipolar expression is selected for its simplicity and quite transparent meaning. Although less practical for numerical calculations than single-ion anisotropy, this spin-pair model is more realistic for describing interfaces and low-symmetry environments. ${ }^{30}$ In Eq. (1), only the pseudodipole term is considered, but it vanishes in cubic symmetry and extension with explicit quadrupole expression would be necessary. ${ }^{25}$ However, at low temperatures, a single crystal of cobalt follows an hep structure. ${ }^{31}$ According to Ref. 32, $K\left(r_{i j}\right)$ is assumed to be a quickly decreasing effective function with distance. Note that the dipole-dipole interaction could also be included in the $K\left(r_{i j}\right)$ function. ${ }^{33}$ The vector $\vec{D}_{i j}$ reflects the local environment of the magnetic moment and can be obtained from first-principles calculations. ${ }^{34}$ In the rest of this paper, because bulk properties are our primarily interest, $\vec{D}_{i j}$ is not considered even though it is usually strong in low-symmetry systems. ${ }^{32}$ Within this spin-pair model of anisotropy, the amplitude of the coupling depends on the interatomic distance and therefore is influenced by the lattice vibrations.

\section{B. Determination of parameters}

The mechanical potential is computed by the embeddedatom method ${ }^{35}$ and parametrized to reproduce bulk Co properties and some of its alloys. ${ }^{36,37}$ Based on the Bethe-Slater curve, the exchange isotropic function $J\left(r_{i j}\right)$ is taken as a continuous function of the atomic distance ${ }^{38}$ as

$$
J\left(r_{i j}\right)=-4 \epsilon\left(-\frac{r^{2}}{\sigma^{2}}+\delta \frac{r^{4}}{\sigma^{4}}\right) e^{-\frac{r^{2}}{\sigma^{2}}} \Theta\left(R_{c}-r_{i j}\right),
$$

where $\Theta\left(R_{c}-r_{i j}\right)$ is the Heaviside step function, $R_{c}$ is a cutoff distance taken to the second and the third nearest neighbor, $\epsilon$ is 


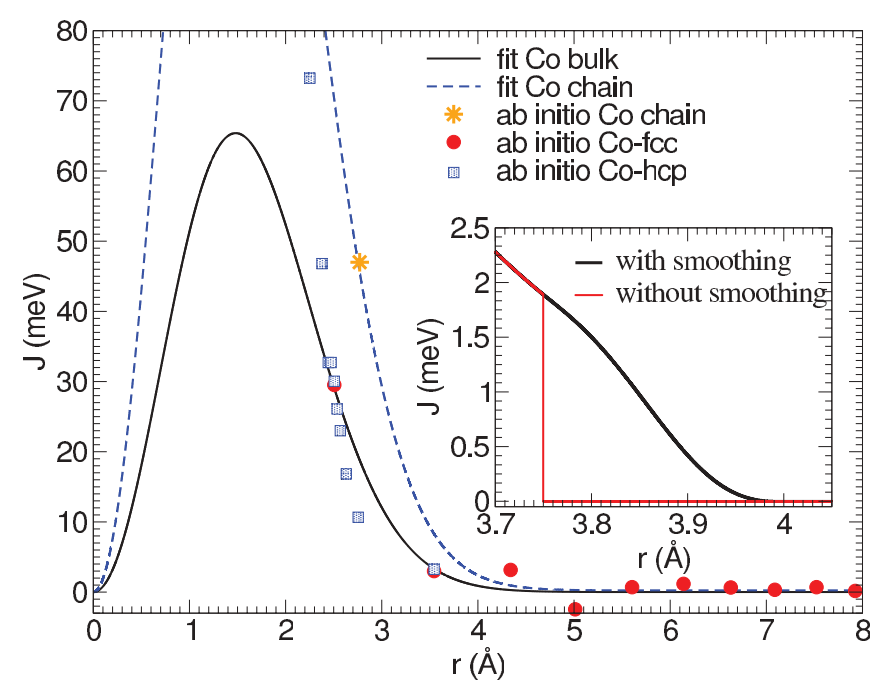

FIG. 2. (Color online) Exchange energy as a function of interatomic distance for cobalt. The solid (black) line shows the $J(r)$ results from a fit on values of exchange integrals [filled (red) circles] obtained from ab initio calculations on hcp $\mathrm{p}^{5}$ and fcc $\mathrm{Co}^{40}$ structures. The dashed (blue) line shows the $J(r)$ results obtained from a rescaling of the previous function such that the rescaled function fits the value of the first-nearest-neighbor exchange interaction $J_{1 \mathrm{NN}}=50 \mathrm{meV}$ estimated for Co clusters deposited on various nonmagnetic substrates. ${ }^{39}$ Considered parameters are for a bulk system, with $\epsilon=44.6928 \mathrm{meV}, \delta=3.496 \times 10^{-3}$, and $\sigma=1.4885$ $\AA$, and for nano-objects (in particular, a one-dimensional chain), with $\epsilon=106 \mathrm{meV}$ and $\delta$ and $\sigma$ unchanged. Inset: The influence of the smooth cutoff function, showing the region between $R_{c}=3.75 \AA$ and $4 \AA$.

the amplitude, $\sigma$ is the spreading distance, and $\delta$ is a parameter allowing a change of sign between antiferromagnetism and ferromagnetism. During the dynamics, discontinuities of the magnetic forces and magnetic energies are observed due to the moving of atoms in and out of the rigid cutoff sphere. Consequently, a fifth-order polynomial tapering function is added in order for the first and second derivatives to go smoothly to 0 at the cutoff radius, as shown in Fig. 2. Because of varying environments, there is no unique set of $a b$ initio exchange functions in cobalt. ${ }^{39}$ So $J$ is determined by fitting various first-principles calculations on the exchange energy of cobalt in fcc, hcp, and one-dimensional structures. ${ }^{40-42}$ Figure 2 shows that first-principles exchange functions are very small from the second neighbors as expected. During the fit $\delta \approx 0$, so whatever the distance, the exchange function in cobalt leads to ferromagnetism. To date no ab initio exchange energy is available for compressed cobalt structures.

The anisotropy function $K$ is more difficult to parametrize. Although the anisotropy energy is often presented as a small quantity, and approximatively several orders of magnitude smaller than the exchange energy, ${ }^{43}$ it is well known that it plays a crucial role in the stabilization of a magnetic state. To our knowledge, there is no attempt to derive an analytical form for this function over the distance for bulk materials from electronic structure calculations. This would require determination of the evolution of the magnetocrystalline anisotropy energy (MCAE) with the interatomic distance, which is not an easy task. Actually the energy difference between an easy and a hard axis is of the order of $10^{-5} \mathrm{eV} /$ atom, which is very difficult to achieve numerically. ${ }^{44}$ Recently, Autès et al. ${ }^{45}$ investigated the MCAE of an iron strand and showed that its evolution with distance can be nonmonotonous and can even change sign. However, this situation is very different from the bulk case and this would probably be hazardous to determine $K$ from such calculations on a one-dimensional chain. A practical application is given in Sec IV.

\section{MOLECULAR AND SPIN DYNAMICS}

\section{A. Microcanonical ensemble: NVE algorithm}

In an isolated physical system in equilibrium the number of particles, volume, and total energy are conserved. By using the dynamical approach, Yang et $_{\text {al. }}{ }^{46}$ were able to generalize the classical Poisson bracket to include spin. Let us consider $f\left(t, \vec{r}_{i}, \vec{p}_{i}, \vec{s}_{i}\right)$ and $g\left(t, \vec{r}_{i}, \vec{p}_{i}, \vec{s}_{i}\right)$, two general functions of time as well as the momenta, coordinates, and spins of each of the particles. The generalized Poisson bracket can be defined as

$$
\{f, g\}=\sum_{i=1}^{N}\left[\frac{\partial f}{\partial \vec{r}_{i}} \cdot \frac{\partial g}{\partial \vec{p}_{i}}-\frac{\partial f}{\partial \vec{p}_{i}} \cdot \frac{\partial g}{\partial \vec{r}_{i}}+\frac{\vec{s}_{i}}{\hbar} \cdot\left(\frac{\partial f}{\partial \vec{s}_{i}} \times \frac{\partial g}{\partial \vec{s}_{i}}\right)\right] .
$$

Such a Poisson bracket is antisymmetric and satisfies Jacobi's identity. This makes the space of smooth functions on a symplectic manifold of an infinite-dimensional Lie algebra, with the Poisson bracket acting as the Lie bracket. This expression applies to any classical system of particles having a Hamiltonian whose variables $X_{i}$ satisfy $\dot{X}_{i}=G\left(X_{i}\right) \frac{\partial \mathcal{H}}{\partial X_{i}}$, where $G$ is an antisymmetric operator acting on variables. Equation (1) satisfies these conditions and the equations of motion can be derived for all the phase-space variables as

$$
\begin{gathered}
\frac{d \vec{r}_{i}}{d t}=\left\{\vec{r}_{i}, \mathcal{H}\right\}=\frac{\vec{p}_{i}}{m_{i}} \\
\frac{d \vec{p}_{i}}{d t}=\left\{\vec{p}_{i}, \mathcal{H}\right\} \\
=\sum_{j(j \neq i)}\left[-\frac{d V\left(r_{i j}\right)}{d r}+\left(\frac{d J\left(r_{i j}\right)}{d r}-\frac{1}{3} \frac{d K\left(r_{i j}\right)}{d r}\right)\left(\vec{s}_{i} \cdot \vec{s}_{j}\right)\right. \\
\left.+\left(\frac{d K\left(r_{i j}\right)}{d r}-\frac{2 K\left(r_{i j}\right)}{r_{i j}}\right)\left(\hat{r}_{i j} \cdot \vec{s}_{i}\right)\left(\hat{r}_{i j} \cdot \vec{s}_{j}\right)\right] \hat{r}_{i j} \\
+\frac{K\left(r_{i j}\right)}{r_{i j}}\left(\left(\hat{r}_{i j} \cdot \vec{s}_{j}\right) \vec{s}_{i}+\left(\hat{r}_{i j} \cdot \vec{s}_{i}\right) \vec{s}_{j}\right), \\
\frac{d \vec{s}_{i}}{d t}=\left\{\vec{s}_{i}, \mathcal{H}\right\}=\vec{s}_{i} \times \vec{\omega}_{i},
\end{gathered}
$$

where $\hat{r}_{i j}$ stands for the normalized vector $\frac{\vec{r}_{i j}}{\left|r_{i j}\right|}$. Up to a certain cutoff radius, the first term in Eq. (5) is the sum of all the spinless mechanical forces followed by additional magnetic forces coming from the varying distance dependence of the exchange and anisotropy energy. Equation (6) is the undamped spin precession equation, where the relation $\left\{s_{i}^{\alpha}, s_{i}^{\beta}\right\}=\epsilon^{\alpha \beta \gamma} s_{i}^{\gamma} \delta_{i j}$ was used for each spin vector component. 
$\vec{\omega}_{i}$ is the effective spin pulsation, which reads as

$$
\begin{aligned}
\vec{\omega}_{i}= & \frac{1}{\hbar} \sum_{j} J\left(r_{i j}\right) \vec{s}_{j}+K\left(r_{i j}\right)\left(\left(\hat{r}_{i j} \cdot \vec{s}_{j}\right) \hat{r}_{i j}-\frac{1}{3} \vec{s}_{j}\right) \\
& +\frac{g \mu_{B} \mu_{0}}{\hbar} \vec{H}_{\mathrm{ext}} .
\end{aligned}
$$

Without the external magnetic field $\vec{H}_{\text {ext }}=\overrightarrow{0}$, taking into account the symmetry, $V\left(r_{i j}\right)=V\left(r_{j i}\right), J\left(r_{i j}\right)=J\left(r_{j i}\right)$, and $K\left(r_{i j}\right)=K\left(r_{j i}\right)$, of interaction potentials, and because of the structure of the equations of motion, it follows that the total energy, the total linear momentum $\vec{P}=\sum_{i} \vec{p}_{i}$, and the total angular momentum $\overrightarrow{\mathcal{J}}=\sum_{i} \vec{r}_{i} \times \vec{p}_{i}+\hbar \vec{s}_{i}$ are conserved quantities over time. In addition, the exact solutions are also time reversible since Eqs. (4)-(6) are invariant with respect to the time inversion transformation $t \rightarrow-t,\left\{\vec{p}_{i}, \vec{s}_{i}\right\} \rightarrow\left\{-\vec{p}_{i},-\vec{s}_{i}\right\}$.

To perform an MMD simulation, all the phase-space variables have to be evolved simultaneously at the same time. In MD, to use a longer time step without loss of short-term accuracy or, alternatively, to achieve a higher accuracy for a given time step, time-reversible or area-preserving symplectic integrators based on ST decompositions have been written from the Liouville formulation of classical mechanics. ${ }^{47,48}$ Later these algorithms were also used in SD simulations of lattice systems. ${ }^{49}$ In the context of spin molecular liquids, Omelyan et al. built algorithms based on the same ST decompositions. ${ }^{20,29}$ Because of the first-order-in-time structure of the system of equations of motion, the superiority of these algorithms compared to predictor-corrector schemes was demonstrated. So, let us consider a set of phase-space variables $\rho\left(\vec{r}_{1} \cdots \vec{r}_{N}, \vec{p}_{1} \cdots \vec{p}_{N}, \vec{s}_{1} \cdots \vec{s}_{N}\right)$ with $3 N$ components, allowing us to write the equations of motion from Eqs. (4)-(6) as

$$
\frac{d \rho(t)}{d t}=\{\rho(t), H\} \equiv\left(L_{r}+L_{p}+L_{s}\right) \rho(t),
$$

with

$$
\begin{aligned}
L_{r} & =\sum_{i=1}^{N} \frac{d \vec{r}_{i}}{d t} \cdot \frac{\partial}{\partial \vec{r}_{i}}, \quad L_{p}=\sum_{i=1}^{N} \frac{d \vec{p}_{i}}{d t} \cdot \frac{\partial}{\partial \vec{p}_{i}}, \\
L_{s} & =\sum_{i=1}^{N} \frac{d \vec{s}_{i}}{d t} \cdot \frac{\partial}{\partial \vec{s}_{i}},
\end{aligned}
$$

where $L_{r}, L_{p}$, and $L_{s}$ are the Liouville operators for position, impulsion, and spin variables, respectively. These operators do not commute with each others because of the radial and spin dependencies due to the interactions. In spite of the fact that Eq. (8) has a formal solution as

$$
\rho(\tau)=e^{\tau\left(L_{r}+L_{p}+L_{s}\right)} \rho(0),
$$

where $\rho(0)$ is a known set of phase-space variables, the exponential propagator cannot be evaluated straightforwardly because $L_{r}, L_{p}$, and $L_{s}$ are noncommuting operators. This requires the use of an ST decomposition of the formal solution to obtain an approximate form. Up to third order in $\tau$,

$$
e^{\tau(A+B+C)}=e^{\frac{\tau}{2} C} e^{\frac{\tau}{2} B} e^{\tau A} e^{\frac{\tau}{2} B} e^{\frac{\tau}{2} C}+\mathcal{O}\left(\tau^{3}\right),
$$

where the choice of ordering $A, B$, and $C$ is arbitrary and noted formally as $(C, B, A, B, C)$. The main advantage of that decomposition is that, in a few simple cases, the formal solution of a single operator is known exactly. It has been shown ${ }^{48}$ that position and velocity operators are simple shift operators, $e^{\tau L_{r}} \vec{r}_{i}(t)=\vec{r}_{i}(t)+\tau \frac{\vec{p}_{i}(t)}{m_{i}}$ and $e^{\tau L_{p}} \vec{p}_{i}(t)=\vec{p}_{i}(t)+\tau \dot{\vec{p}}_{i}(t)$, and that these exponential operators are locally exact. Moreover, because of the commutative relations between nonoverlapping generalized coordinates, $e^{\tau L_{r}}=\prod_{i=1}^{N} e^{\tau L_{r_{i}}}$ and $e^{\tau L_{p}}=$ $\prod_{i=1}^{N} e^{\tau L_{p_{i}}}$. In the case of the spin, it has been shown ${ }^{20,29,49,51}$ that the rotation of an individual spin around an axial vector can be approximated by a third-order expansion in time as

$$
\begin{aligned}
e^{\tau L_{s_{i}}} \vec{s}_{i}= & \frac{\vec{s}_{i}+\tau\left(\vec{\omega}_{i} \times \vec{s}_{i}\right)+\frac{\tau^{2}}{2}\left[\left(\vec{\omega}_{i} \cdot \vec{s}_{i}\right) \vec{\omega}_{i}-\frac{1}{2}\left(\vec{\omega}_{i} \cdot \vec{\omega}_{i}\right) \vec{s}_{i}\right]}{1+\frac{\tau^{2}}{4} \vec{\omega}_{i} \cdot \vec{\omega}_{i}} \\
& +\mathcal{O}\left(\tau^{3}\right),
\end{aligned}
$$

which is a norm-preserving operation for each spin. Then, because the different partial components of $L_{S_{i}}$ do not commute with one another, one has to operate an ST decomposition for the total spin operator $e^{\tau L_{s}}=$ $e^{\frac{\tau}{2} L_{s_{1}}} \cdots e^{\frac{\tau}{2} L_{s_{N-1}}} e^{\tau L_{s_{N}}} e^{\frac{\tau}{2} L_{s_{N-1}}} \cdots e^{\frac{\tau}{2} L_{s_{1}}}+\mathcal{O}\left(\tau^{3}\right)$ up to third order in time to be self-consistent with Eq. (12). In order to improve consistently the reliability of the proposed integration, extended third-order and higher order decomposition schemes can be implemented and appear to be more efficient, despite the usage of a larger number of exponential propagators. ${ }^{19}$

As a consequence, the choice of ordering of the operators in Eq. (11) is dictated by the amplitude of the forces and effective pulsations. Let $\mathrm{R}, \mathrm{P}$, and $\mathrm{S}$ be the evolution operators of position, impulsion, and spin, respectively. Several time orderings of operators have been proposed in the literature. References 20 and 29 suggest the (P,R,S,R,P) ordering, Ref. 49 explores (S,P,R,P,S), and Ref. 26 quotes (S,R,P,R,S). Numerically one has to put the most computer-time-consuming operator for A in Eq. (11), because it has to be evaluated once. In our case, the shortest time step is given by the amplitude of the effective pulsation, and for the values given in Fig. 2, one has to keep $\tau \omega_{i} \leqslant 0.1$. This means that because of the typical amplitude of the exchange interaction, a time step smaller than the usual MD time step has to be imposed (about 0.2 vs $1 \mathrm{fs}$ ) but has to be carefully compared to the amplitude of mechanical forces. ${ }^{52}$

Algorithms have to be classified according to their computational speed and accuracy. As an example, simulations with 1342 atoms of cobalt in an fcc structure with periodic boundary conditions at equilibrium volume during 5 ps are performed. The first simulation is run by considering initial velocities corresponding to a kinetic temperature of $1 \mathrm{~K}$ [see Fig. 3(a)] in a perfect ferromagnet. The second simulation is run with a small random rotation done for a single spin at $t=0$ without the initial mechanical speed [see Fig. 3(b)]. Before analyzing the energy conservations between these two situations, let us compare their execution times (see Table I). The $(\mathrm{P}, \mathrm{R}, \mathrm{S}, \mathrm{P}, \mathrm{R})$ algorithm is the fastest since the spin evolution operator appears only once. The time evolution of the total energy is shown in Fig. 3 and the accuracy depends on the choice of the algorithm but is also linked to the nature of the excitation. In the first case, the spins do not evolve because the system remains perfectly ferromagnetic. This situation is then identical to the comparison of accuracy between position-Verlet and velocity-Verlet algorithms, which favors 

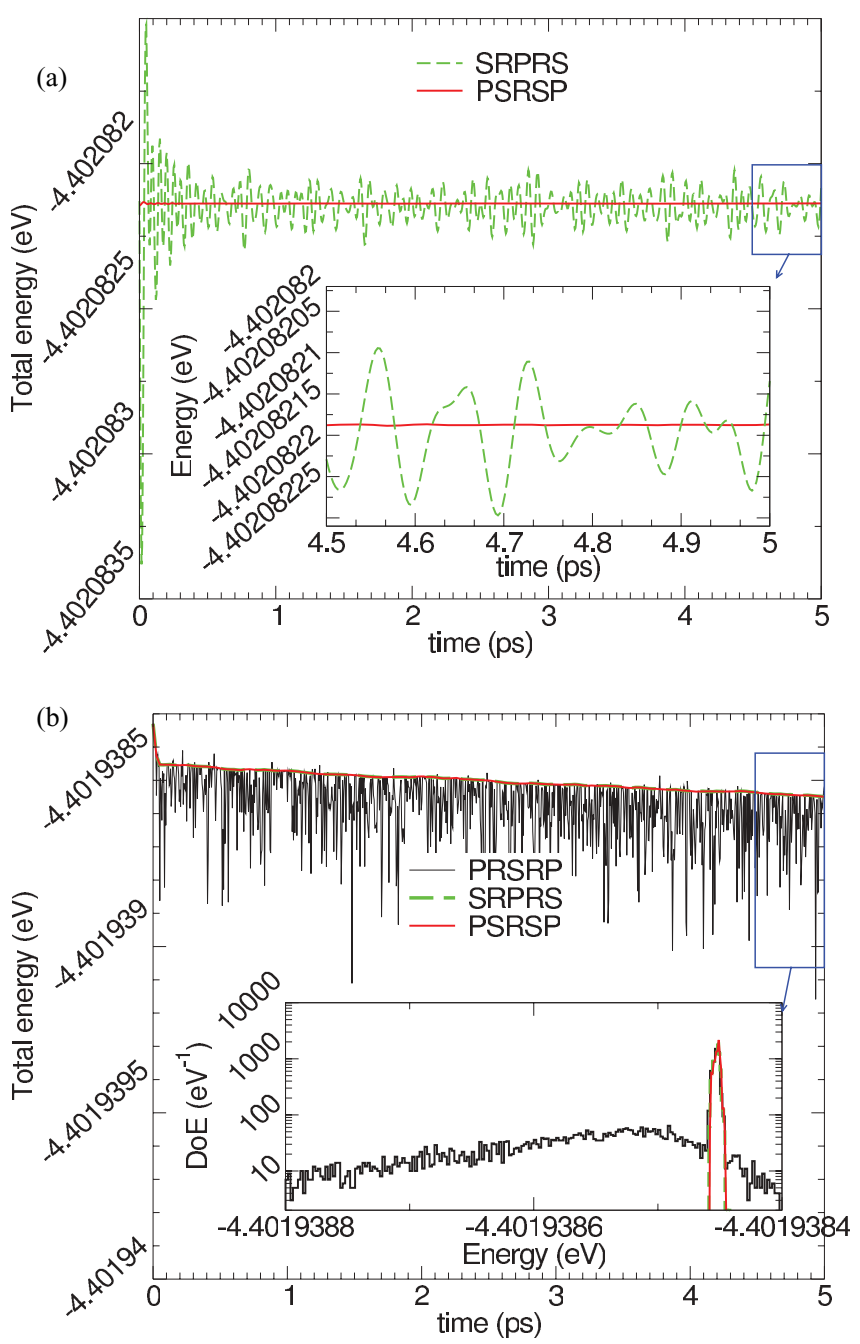

FIG. 3. (Color online) Total energy of a system of 1342 atoms of cobalt in the fcc configuration. (a) A pure ferromagnet with a mechanical perturbation is applied at $t=0$, corresponding to $T=$ $1 \mathrm{~K}$. (b) Without the initial kinetic velocity, one spin is randomly moved at $t=0$. Inset (a): A zoom-in on the variations of energy. Inset (b): Density of states between all algorithms, revealing the spreading of the PRSRP method.

the velocity-Verlet situation. ${ }^{48}$ It is also known that the velocity-Verlet algorithm is area preserving, is time reversible, and provides the absence of long-term energy drift. In the second situation, the spins rotate around their effective axis and provide the dominating dynamics of the whole system because the kinetic temperature is nearly 0 . In this situation $(\mathrm{P}, \mathrm{R}, \mathrm{S}, \mathrm{R}, \mathrm{P})$ remains the fastest but less accurate algorithm. $(\mathrm{P}, \mathrm{S}, \mathrm{R}, \mathrm{S}, \mathrm{P})$ is the most accurate algorithm because the effective pulsation is evaluated twice and the forces only once. This

TABLE I. Relative speeds to (P,R,S,R,P) algorithm of three versions of the Suzuki-Trotter decomposition scheme.

\begin{tabular}{cccc}
\hline \hline & $(\mathrm{P}, \mathrm{R}, \mathrm{S}, \mathrm{R}, \mathrm{P})$ & $(\mathrm{P}, \mathrm{S}, \mathrm{R}, \mathrm{S}, \mathrm{P})$ & $(\mathrm{S}, \mathrm{R}, \mathrm{P}, \mathrm{R}, \mathrm{S})$ \\
\hline $\mathrm{P}, \mathrm{R}, \mathrm{S}, \mathrm{R}, \mathrm{P})$ & 1 & 1.41 & 1.45 \\
\hline \hline
\end{tabular}

algorithm has the same level of accuracy as (S,P,R,P,S) considered in Ref. 49, but in that case, the effective pulsation and the forces are evaluated twice, which is much more time-consuming. Surprisingly, the (S,R,P,R,S) algorithm can be excluded since it is less accurate from the mechanical point of view and time-consuming from the spin point of view. The spin pseudo-Hamiltonian cannot be rigorously conserved and its difference from the true spin Hamiltonian remains due to the spin evolution algorithm. This is why there is a long-term drift in the energy, which can be systematically controlled either by reducing the time-step value or by increasing the order of the spin evolution operators. ${ }^{18,19,53}$ In that closed microcanonical system, each component of the total angular momentum of an isotropic Heisenberg model is conserved. Thus starting from a zero-total-magnetic-moment situation, the spin system stays in its paramagnetic-like configuration over time. When an anisotropy axis is considered in a more general anisotropic exchange situation, only the component of the total spin moment along this axis is conserved. ${ }^{20}$ In decomposition algorithms, exact conservation of the total energy and total angular momentum cannot be achieved. This has been proved to be important for spin liquids near phase transitions, where the presence of artificial fluctuations in energy may have a significant influence on the results. ${ }^{54}$ However, in most applications below the Curie point, the accuracy achieved by such decomposition algorithms is high enough to obtain reliable results.

\section{B. Canonical ensemble: NVT algorithm}

Temperature is a fundamental quantity in statistical physics and its microcanonical value is usually evaluated in standard thermodynamics by differentiating entropy with respect to the internal energy of the system. ${ }^{55}$ Since Maxwell in spinless $\mathrm{MD}$, the temperature is probed by monitoring the average kinetic energy of atoms assuming equivalence of ensembles and ergodicity.

The thermal equilibration of a closed system of interacting atomic spins is fundamentally different from that of a closed system of interacting atoms. ${ }^{56}$ In a mechanical system without internal degrees of freedom, because of the virial theorem and in the thermodynamical limit, the pressure can be found for a given microcanonical temperature. This provides a convenient way for spinless systems to reach equilibrium. There is no such equivalence for a pure spin system. However, imposing dissipation on the evolution equations leads to a nonconserving total energy situation, and hence a canonical ensemble (at least a subset, because of the periodic boundary conditions) can be approached with a microcanonical ensemble. An exchange of energy between a finite system and a thermostat causes fluctuations in the thermodynamical properties. Coupling this $N$-particle system to a heat bath of constant temperature and infinite energy allows a canonical ensemble to capture these energy fluctuations.

The rescaling, deterministic, and stochastic approaches are generally used in MD to generate a canonical ensemble, ${ }^{48}$ and they have been partially extended to molecular spin systems. The first method involves direct control of the kinetic energy. The velocity of each particle is periodically rescaled such that the kinetic energy instantaneously matches the 
desired temperature. ${ }^{57}$ Although it is a popular approach, the rescaling method does not produce trajectories that lead to a canonical ensemble. The isokinetic MD extension scheme of Evans and Morris yields correct canonical ensemble averages only for properties depending on the positions of the particles. ${ }^{48}$ However, no derivation for molecular spin systems has been established so far.

In the deterministic approach, an extended Hamiltonian with auxiliary degrees of freedom connected to a heat bath is formed $^{58}$ to control the temperature using continuous dynamics. In pure MD, this approach was unified by Nosé ${ }^{59}$ in the formulation of Hoover. ${ }^{60}$ This extended system is ergodic and generates distributed phase-space trajectories correctly. This way of thermostating the temperature was also applied to a spin system but was rarely tested in realistic spin dynamics. ${ }^{61,62}$ Nevertheless, accelerated simulations of large systems have recently been investigated and proven to be competitive with the stochastic method. ${ }^{63}$

In the stochastic approach, the particles are subject to some random process which alters their momenta. The best-known example of this method is the stochastic Langevin equation for Brownian motion applicable in both $\mathrm{MD}^{48}$ and atomic spin dynamics. ${ }^{64,65}$ According to Andersen, ${ }^{58}$ each particle is forced to undergo a fictitious collision in which a new velocity is assigned from the Maxwell-Boltzmann distribution. Although the trajectories are discontinuous and irreversible as in the rescaling method, this remains the easiest method for performing simulations in a canonical ensemble since it is very simple to implement numerically. However, in atomic spin dynamics, the stochastic Landau-Lifshitz-Gilbert equation with multiplicative noncommutative noise has to be solved. According to the numerical scheme used, this conducts different Fokker-Planck equations for the average probability density depending on the stochastic sense chosen, which may converge inconsistently with the proper thermal-equilibrium properties. ${ }^{66}$

The coupling between the lattice and the spins is driven by the spatial dependence of the exchange and magnetic anisotropy. Control of the temperature of one of the two subsystems should allow the other subsystem to thermalize towards the same equilibrium temperature. According to the energy balance, the total energy of the whole system is not a constant in time, and a partial temperature of each subsystem is a measure of the corresponding entropy production. While it is common to get the temperature of a mechanical spinless subsystem from the average kinetic energy, the corresponding spin temperature has to be defined and followed in time during the simulations. According to Nurdin et al. ${ }^{67}$ for a large number of atoms $N$, one has

$$
k_{B} T_{S} \approx \frac{\sum_{i=1}^{N}\left|\vec{s}_{i} \times \vec{\nabla}_{i} \mathcal{H}\right|^{2}}{\sum_{i=1}^{N}\left[\vec{s}_{i} \times \vec{\nabla}_{i}\right] \cdot\left[\vec{s}_{i} \times \vec{\nabla}_{i}\right] \mathcal{H}},
$$

where $\mathcal{H}$ is the Hamiltonian of the system and $\vec{\nabla}_{i}=\frac{\partial}{\partial \vec{s}_{i}}$ is the gradient along the spin direction. Using Eq. (1), the spin temperature can be written as

$$
T_{S}=\frac{\hbar}{2 k_{B}} \frac{\sum_{i=1}^{N}\left|\vec{s}_{i} \times \vec{\omega}_{i}\right|^{2}}{\sum_{i=1}^{N} \vec{s}_{i} \cdot \vec{\omega}_{i}},
$$

where $\vec{\omega}_{i}=-\frac{1}{\hbar} \frac{\partial H}{\partial \vec{s}_{i}}$ is the effective pulsation for atom $i$. A stochastic method is developed to generate a canonical ensemble in our MMD. The temperature is controlled stochastically by using both a dissipation and a fluctuation in the magnetic subsystem only. The stochastic Landau-LifshitzGilbert equation is

$$
\frac{d \vec{s}_{i}}{d t}=\frac{1}{1+\alpha^{2}}\left[\vec{s}_{i} \times\left(\vec{\omega}_{i}+\overrightarrow{\tilde{\omega}}_{i}(t)\right)+\alpha \vec{s}_{i} \times\left(\left(\vec{\omega}_{i}+\overrightarrow{\tilde{\omega}}_{i}(t)\right) \times \vec{s}_{i}\right)\right],
$$

where $\overrightarrow{\tilde{\omega}}_{i}(t)$ is a random pulsation which satisfies $\left\langle\tilde{\omega}_{i a}(t) \tilde{\omega}_{j b}(t)\right\rangle=2 D \delta_{i j} \delta_{a b} \delta\left(t-t^{\prime}\right)$ and $\alpha$ a dimensionless damping constant. $D$ is the amplitude of the correlation noise and is deduced from the Fokker-Planck equation for the whole MMD set of equations (4)-(6), where Eq. (6) is substituted by Eq. (15). Because of our Poisson bracket definition, the derivation of the Fokker-Planck equation in the calculus of Stratonovich gives a vanishing divergence of the mechanical phase-space velocity. This reduces to a calculation of $D$ identical to the nonlinear Langevin equation of the spin subsystem alone, which gives $D=\alpha k_{B} T / \hbar$.

An MMD simulation on an hep Co is performed to follow both the instantaneous average translational temperature $T_{L}=\frac{1}{3(N-1) k_{B}} \sum_{i=1}^{N} \frac{\vec{p}_{i}^{2}}{m_{i}}$ and the spin temperature $T_{S}$. In this simulation, $T_{L}$ is not controlled by any method and the velocities are not rescaled to match the target temperature. All spins are initially aligned along the $c$ axis, while the initial velocity on atoms is 0 . The target temperature $T=600 \mathrm{~K}$, below the Curie temperature, and the lattice, impulsions, and spins are free to move according to their corresponding equations of motion. The temperature of the two subsystems is followed in Fig. 4(a), while the time evolution of the average magnetization is presented in Fig. 4(b). As expected, the coordinate dependence of the exchange interaction and anisotropy functions allows equilibration between $T_{L}$ and $T_{S}$. The lattice is heated up to $0.2 \mathrm{~ns}$, while the average magnetization direction quickly follows the mean-field state for a $600 \mathrm{~K}$ temperature according to the Curie law. All
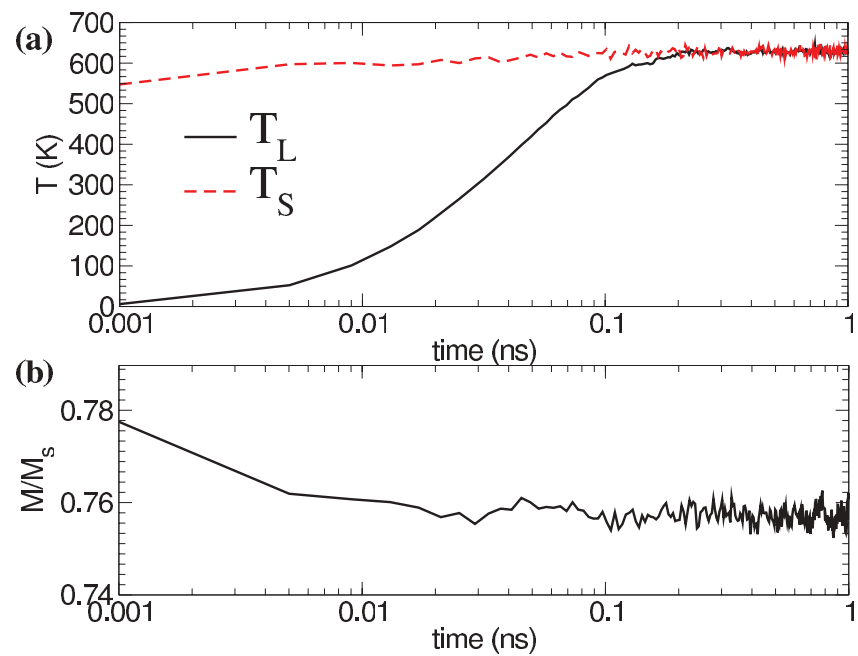

FIG. 4. (Color online) MMD simulation of an hcp Co illustrating the coupling between the lattice and the magnetic subsystems. 
the temperatures converge in a unique canonical temperature with the desired magnetic state. The same conclusion was derived recently in Ref. 65 without consideration of the whole nonlinear set of Langevin equations.

\section{APPLICATIONS}

Let us illustrate our MMD algorithm with two examples involving cobalt nanowires. The first example is a free-standing (i.e., unsupported) monatomic cobalt wire, and the second example is a finite wire deposited along the step of a Pt surface. Because both situations consider cobalt-based nanostructures, one has to pay attention to the strength of the interaction potential with respect to its bulk parametrization. In particular, it is known that the magnetic interaction is reinforced in low dimensions ${ }^{39}$ and the exchange interaction parameter given in Fig. 2 has to be slightly rescaled. Moreover, the determination of the interatomic dependence of the anisotropy strength $K(r)$ in these systems is presented.

\section{A. An ab initio infinite isolated cobalt nanowire}

Autès et al. have shown that the magnetic anisotropy of an iron nanowire changes rapidly with the interatomic distance. $^{45}$ In particular, a switch of the easy axis from parallel to perpendicular to the wire axis was obtained under compression. For a single infinite cobalt nanowire, similar calculations have been performed using the density functional theory code QUANTUM ESPRESSO ${ }^{68}$ which is based on a planewave expansion of the electronic density of full-relativistic pseudopotentials. The simulation box considered is made of a single atom with periodic boundary conditions. The periodicity along two directions has been taken large enough $(\sim 15 \AA)$ to avoid any interaction between adjacent wires. Along the wire axis, the period is varied between 2 and $7 \AA$. The calculations have been performed within the LDA using the Perdew-Zunger exchange-correlation parametrization. The plane-wave energy cutoff was taken as 35 Ryd for the wave function and 250 Ryd for the charge density. More than $500 k$ points supplemented with a Gaussian smearing have been used to integrate over the whole Brillouin zone, which has ben proved sufficient to ensure keeping the precision of the MCAE calculation below $0.01 \mathrm{meV}$.

As already shown for an iron wire, ${ }^{45}$ a uniaxial expansion of the MCAE, $E(\theta)-E(0)=K_{1} \sin ^{2} \theta$, is also an excellent approximation for an unsupported cobalt wire (see inset in Fig. 5) along the considered distances. $K$ is directly obtained by the MCAE, if one neglects the second-nearest-neighbor interactions (which is reasonable for a wire). Thus for a considered distance $d, K(d)=K_{1}(d) / 2$, where the factor 2 originates from the fact that each atom has two neighbors in the first shell. Note that, as for Fe, the MCAE of an infinite cobalt wire (see Fig. 5) presents a change of sign. At a distance of $2.42 \AA$, the axis along the wire switches from the difficult to the easy magnetization axis (and vice versa). For distances above this value the magnetization tends to align along the direction of the wire, while for smaller distances a perpendicular alignment is preferred. Interestingly, around $3.2 \AA$, the MCAE shows a rather narrow maximum and values as large as $8 \mathrm{meV}$. The MCAE curve has been fitted by a

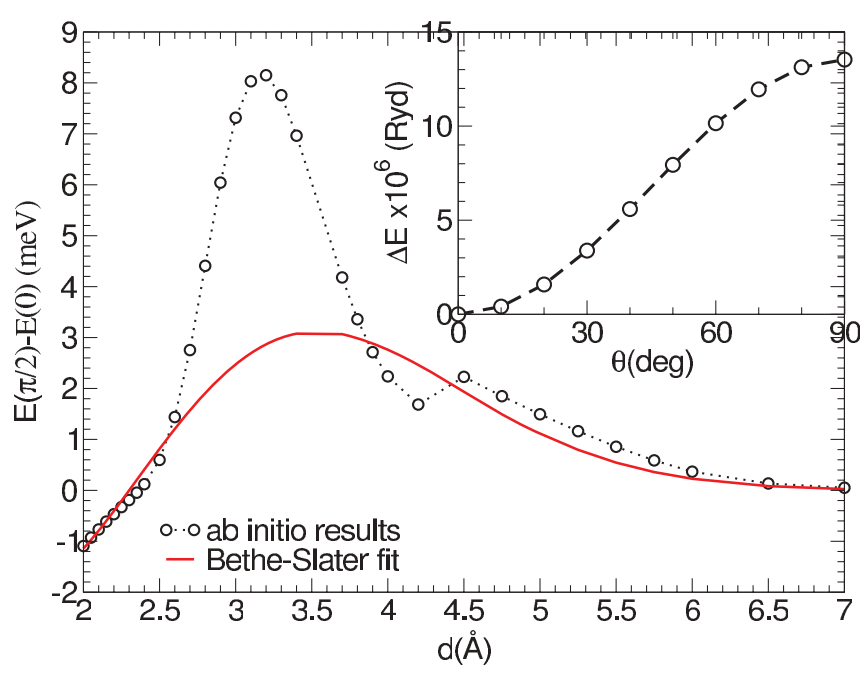

FIG. 5. (Color online) Variation of the MCAE of an infinite cobalt wire as a function of the interatomic distance (circles) obtained from $a b$ initio calculations. Positive values of the MCAE correspond to an easy axis parallel to the wire. An anisotropy function $K(r)$ of analytical form from Eq. (2) is fitted to the $a b$ initio data and is represented in by the solid (red) line. The parameters of this anisotropy function are $\epsilon=-0.00330282 \mathrm{eV}, \delta=0.864159$, and $\sigma=2.13731 \AA$. Inset: One MCAE angular variation for $d=2.5 \AA$ and its $\sin ^{2}(\theta)$ fit.

Bethe-Slater function. Even though the fit is not perfect, it correctly reproduces the general trend and the slope around the equilibrium distance of $d=2.5 \AA$ obtained in bulk cobalt.

\section{B. A finite isolated cobalt nanowire}

Using these parameters, MMD simulations have been performed for a free chain of 20 atoms aligned in the $z$ direction with periodic boundary conditions. An initial lattice spacing of $d=2.4 \AA$, which represents the equilibrium lattice spacing for such a chain, is considered such that the system will fluctuate around its equilibrium state. The spin directions are initially random and all the atoms have an initial velocity taken from a distribution to a temperature equal to $200 \mathrm{~K}$. The magnetic damping $\alpha$ is equal to 0 . The total energy is decomposed into three components: the potential, the kinetic, and the magnetic energies. For such an isolated NVE system, the sum of these three components is conserved in time at the order of the algorithm, as shown in Fig. 6(b). At less than 5 ps, one observes a stabilization of each component of the energy. The system is in both magnetic and mechanical equilibrium. Before this stabilization, a relaxation process is observed, with an increase in the mechanical and magnetic energy, which is associated with the global cooling of the system.

Another situation is now considered by varying the interatomic distances from 2.2 to $2.8 \AA$, where the average magnetization vector components are followed in time. During these calculations, the ionic degrees of freedom is frozen and only the spins are allowed to move. All the spins are initially perpendicular to the wire. The target temperature is kept at 0 but a finite magnetic damping constant of $\alpha=0.1$ is considered. The time evolution of the magnetization along the wire ( $z$ axis) and perpendicular to it ( $x$ axis) are shown in 


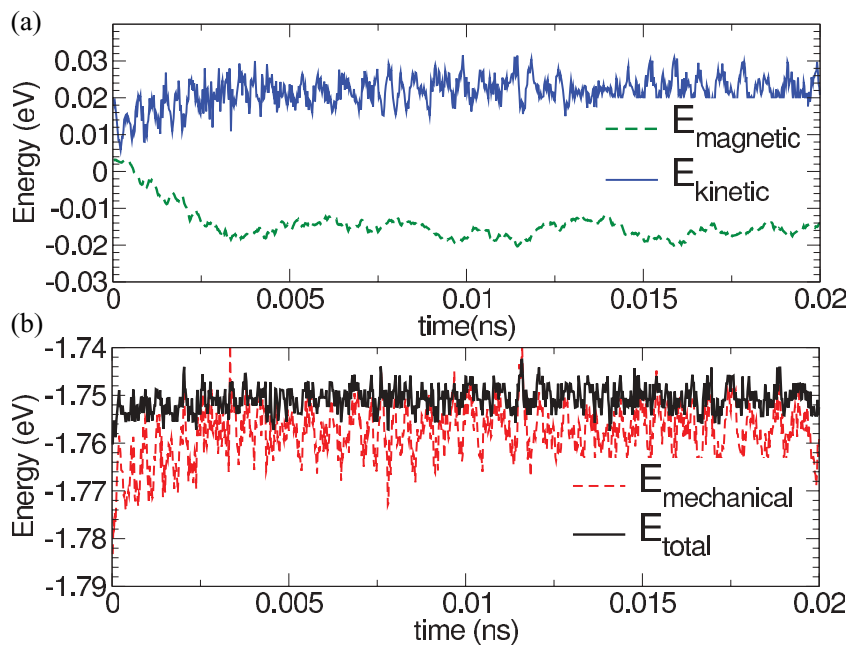

FIG. 6. (Color online) Energy decomposition of a 20-atom linear chain separated by $d=2.40 \AA$ and periodic boundary conditions. Spin directions are random for $t=0$ and $\alpha=0$. Initial impulses are taken from a distribution with a temperature of 200 K. (a) Kinetic and magnetic energies; (b) total and mechanical energies

Fig. 7. The inversion of sign of the anisotropy which appears at $2.42 \AA$ is clearly visible in the behavior of the magnetization. For an interatomic distance below this threshold, the easy axis is perpendicular to the wire and the spins remain quenched in their initial orientation. For interatomic distances larger than this threshold, a reorientation is observed. In the particular case of a distance $d=2.50 \AA$, the anisotropy is so small (see Fig. 5) that it takes more than a nanosecond to switch to the easy axis.

\section{Finite cobalt wire on a (111)Pt substrate}

Recently controlled growth and characterization of magnetic chains on nonmagnetic crystal surfaces have been made possible by new advances in experimental techniques. Such
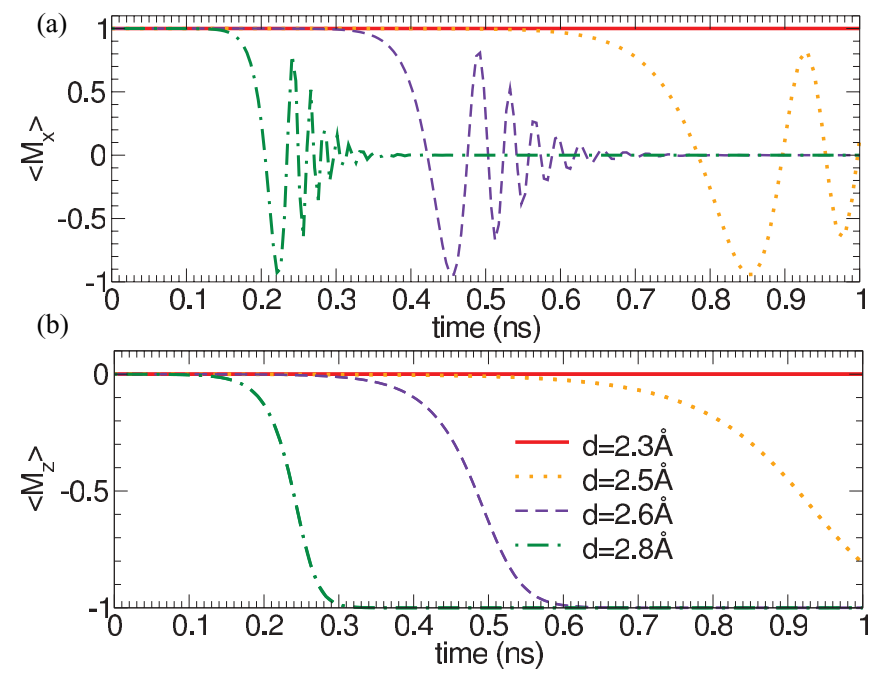

FIG. 7. (Color online) Magnetization behavior of a 20-atom cobalt chain: (a) $x$ component and (b) $z$ component of the average magnetization for four lattice spacings. Simulations were performed with exchange and anisotropy interactions with the same parameters as presented in Figs. 2 and 5, including $\alpha=0.1$ and $T=0$.

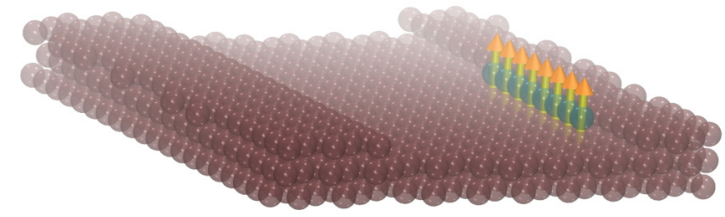

FIG. 8. (Color online) Snapshot of a monatomic chain of eight atoms of Co deposited on a (111)Pt substrate.

chains, a few nanometers long, are quite stable due to their growth at terrace step edges. ${ }^{69}$ The control of magnetocrystalline anisotropy at this scale makes it possible for information storage to overcome the critical superparamagnetic limit. A finite-length chain of classical magnetic moment has been widely studied both analytically using the Stoner-Wohlfarth model $^{64,70}$ and numerically using atomistic spin dynamics calculations. ${ }^{39,71}$

MMD simulations have been performed to study the time evolution of the average magnetization of varying length of monatomic cobalt chains deposited on a (111)Pt substrate. The chains decorate a terrace step edge along the $y$ axis according to Fig. 8. The substrate is composed of 980 atoms of platinum which are considered to be nonmagnetic. The mechanical potential between all pairs is given in Ref. 37. The size of the studied nanowires ranges from 4 to 74 atoms of Co. Although the substrate is periodically repeated in the $x$ and $z$ directions, the cobalt chains remain isolated from each other. The parametrization of the mechanical potential was presented in Sec. II B. The exchange energy interaction $J(r)$ has been rescaled such that its numerical value is $J_{1}=50 \mathrm{meV}$ for a lattice spacing $d=2.77 \AA$, corresponding to the first-nearestneighbor distance of $\mathrm{Pt}$. The magnetic anisotropy function has to be parametrized carefully since it is well known that Pt plays a crucial role in the overall anisotropy of the wire. Experimentally, for a single Co chain on the step -edge of a $\mathrm{Pt}(111)$ surface, the easy axis was found to be perpendicular to the wire direction, forming an angle of $43^{\circ}$ with the terrace normal pointing in the direction of the upper terrace, ${ }^{69}$ and the MCAE was as large as $2 \mathrm{meV}$. This result was also found by $a b$ initio calculations. ${ }^{72}$ Without considering an extra single axis pointing in this direction, the simple analytical form of Eq. (1) is unable to reproduce such complex anisotropic behavior if nonmagnetic platinum atoms are considered. Since our MCAE found for the unsupported chain at $d=2.77 \AA$ is also of the order of $2 \mathrm{meV}$ with an easy axis along the chain, the sign of $\epsilon$ in the Bethe-Slater function of anisotropy is reversed to get the easy axis off the wire. Since the interatomic distance between atoms in the chain fluctuates a bit, the exact form of $K(r)$ is not very relevant. As the Pt-Co interaction is characterized by a strong spin-orbit coupling, a magnetic damping parameter of $\alpha=0.1$ was chosen.

The MMD simulation data obtained for a chain of $L=8$ atoms are shown in Fig. 9 and analyzed using random telegraph noise analysis in the time domain. ${ }^{73}$ All magnetic moments are initially along the easy axis. For $T=75 \mathrm{~K}$, after an average waiting time of $5 \mathrm{ps}$, a rapid reversal event occurs, followed by frequent switches between $M_{z}= \pm 0.85$. The total simulation length is selected to obtain a minimal number of 40 switching events. For the lowest considered temperature, the simulation 

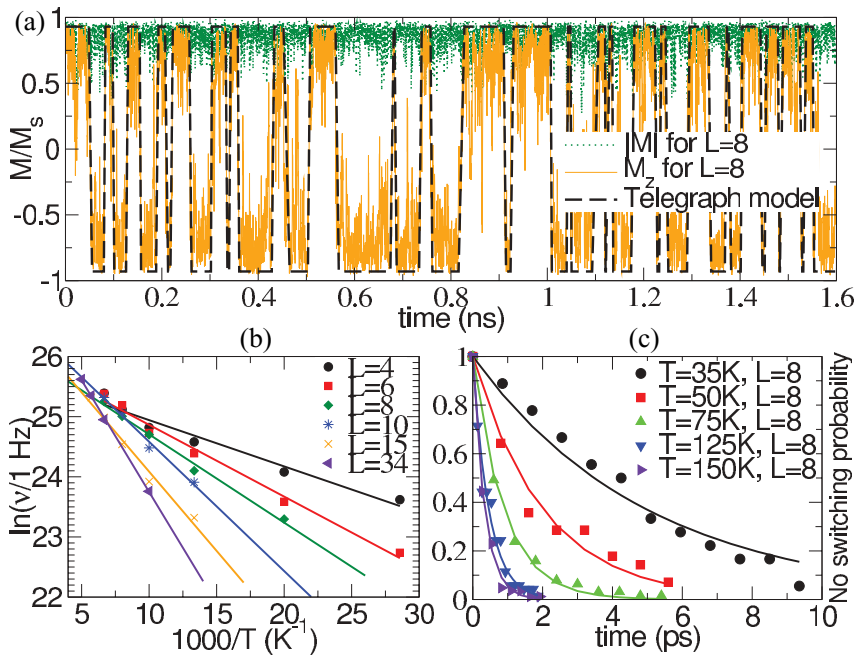

FIG. 9. (Color online) (a) Time fluctuations of both $z$ components (with respect to the norm) of the average magnetization for a chain of $L=8$ atoms at $75 \mathrm{~K}$ [orange, with respect to green]; the dashed (black) line is from the so-called two-state random telegraph noise analysis. ${ }^{73}$ (b) Logarithm of the frequency of reversal versus the temperature, plotted for varying lengths. Solid lines correspond to the linear fit. (c) Probability of nonswitching versus waiting time, plotted for five temperatures and for a chain of eight Co atoms. Lines correspond to the exponential decay fit.

time runs up to $4 \mathrm{~ns}$. Analysis of these fluctuations by the threshold two-state telegraph model allows the determination of the frequency of reversal for each temperature and for various chain lengths. An approximate thermal activation law is given by $v=v_{0} \exp \left(E_{a} / k_{B} T\right)$, where $v_{0}$ is not a simple constant but a complicated function which depends on the system size, temperature, and anisotropies. However, this law has already been proven reasonable for extraction of the activation energy $E_{a} \cdot{ }^{74,75}$ The logarithm of the frequency

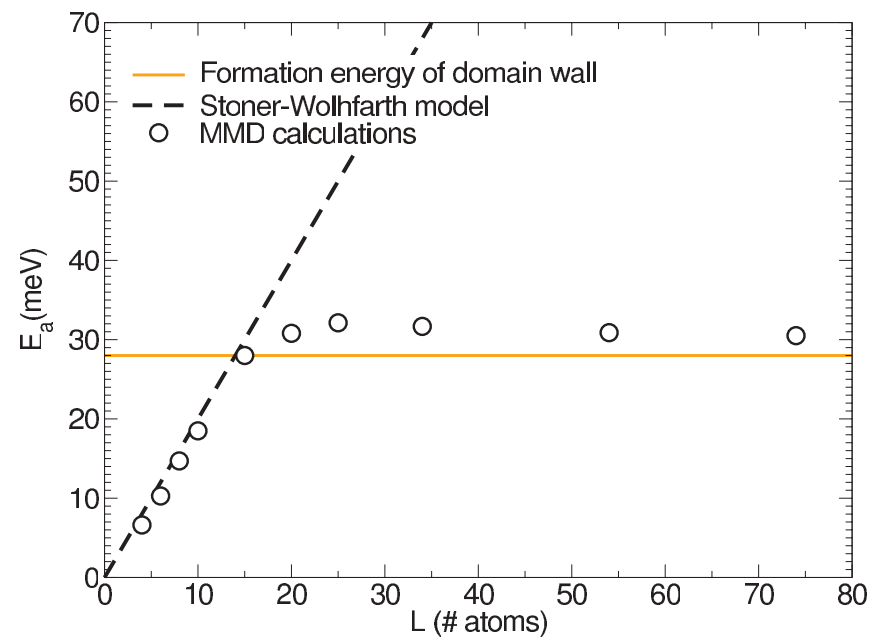

FIG. 10. (Color online) Activation energy versus number of atoms from MMD calculations (circles) and from the StonerWolhfrath model, where the chain magnetization is approximated by a single macrospin of rigid magnitude [dashed (black) line]. The horizontal (orange) line is the minimal energy for the formation of a domain wall given by Ref. $7(E=2 \sqrt{2 J K})$.

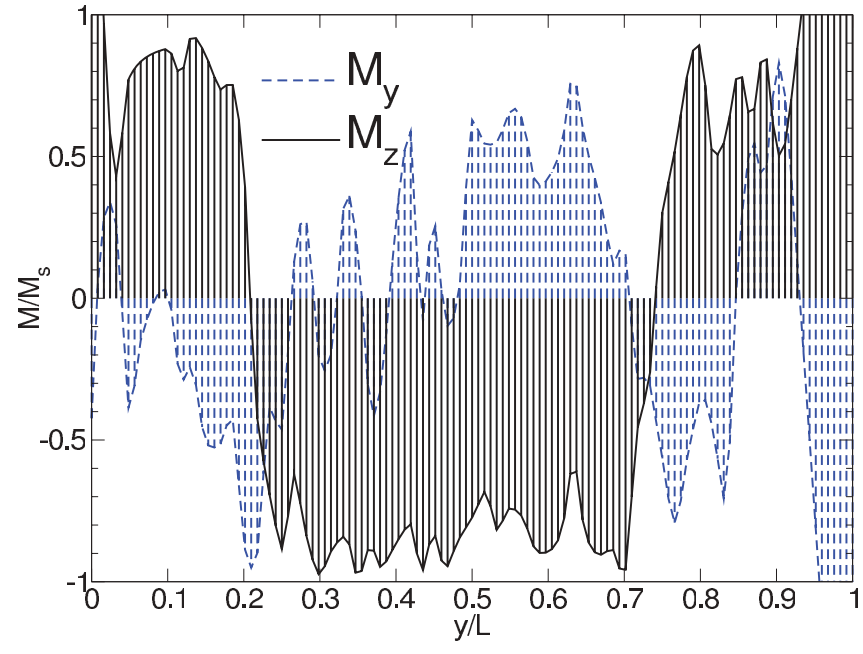

FIG. 11. (Color online) Transversal and longitudinal components of the magnetization for a chain of $54 \mathrm{Co}$ atoms after a nucleation time corresponding to the zero component of the average magnetization along the easy axis.

versus the inverse of the temperature can be plotted to extract the activation energy from the slope of the linear fit. The activation energy curve in Fig. 10 is evidence of the domain wall nucleation mechanism in the chain. Up to a characteristic length $L_{D} \approx 15$, extracted beyond where the creation of a magnetic domain wall is favorable, the MMD simulations are in agreement with the Stoner-Wohlfarth model, which predicts an increase in the activation energy proportional to the total anisotropic energy. The energy of the creation of a Bloch wall can be obtained analytically in the limit of infinite length and takes the simple form $E=2 \sqrt{2 J K}$. Comparing this energy to the value of the coherent reversal gives $L_{D}=2 \sqrt{2 J / K}$. With the numerical values of $J=50 \mathrm{meV}$ and $K=2 \mathrm{meV}$, one immediately gets $L_{D} \approx 14$ (in units of lattice spacing) and $E \approx 28.3 \mathrm{meV}$. These values are in very good agreement with the results found in our MMD simulations. Beyond $L_{D}$, the activation energy goes to the formation energy of a domain

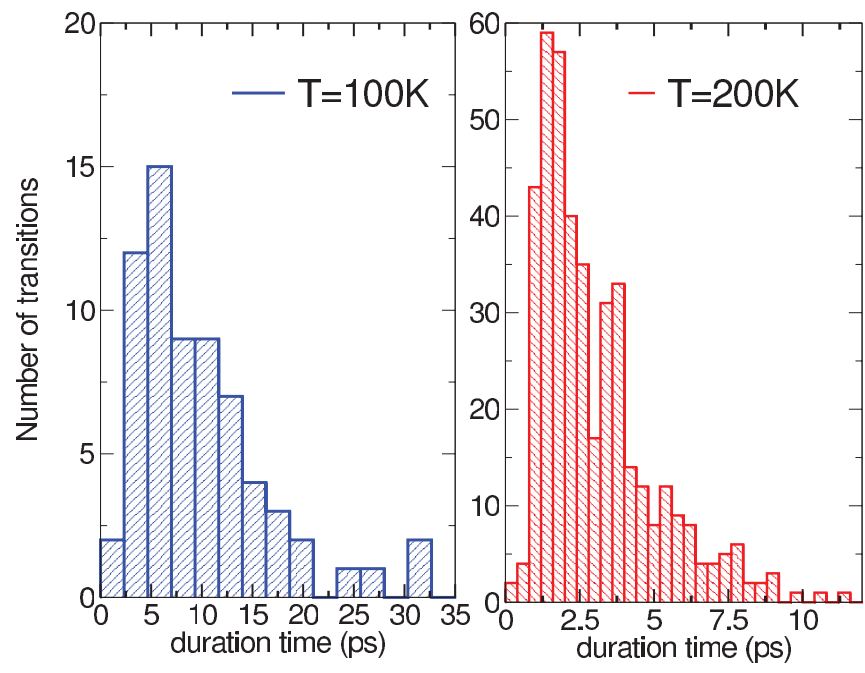

FIG. 12. (Color online) Histograms of the switching time of a 54-atom chain during a simulation of $3.2 \mathrm{~ns}$ at two temperatures. 
wall. Our results agree with the scenario described in Ref. 71: up to $L_{D}$, all magnetic moments are in coherent rotation; above this length the activation energy has an interesting $1 / L$ dependency during the single-nucleus phase. For a longer chain with multiple nucleations, the prefactor of the thermal activation law no longer depends on $L$.

Figure 11 shows a snapshot of the magnetization profile of a chain of $L=54$ atoms during a reversal process obtained after a time when $M_{z}=0.0$. Although not depicted, the same type of profile shows a coherent rotation of all magnetic moments for $L<15$, whereas a single domain wall starts to appear for $20 \leqslant$ $L \leqslant 35$. Aanalysis of the transition period between a single reversal reveals a complex situation with fast domain wall motion. For the range of temperature considered and for chains 54 atoms long, the average time of the first magnetization switch falls between 2 and 7 ps (see Fig. 12).

\section{CONCLUSION}

To summarize, an MMD model including an explicit spinpair magnetic anisotropy coupling between localized magnetic moments and mechanical displacements at finite temperatures is derived. A parametrization from $a b$ initio calculations of this pair anisotropy is given to obtain the magnetic behavior of monatomic chains of cobalt deposited on a (111)Pt substrate. On a moving lattice, this magnetic pair model reproduces previous magnetic switching mechanisms without considering a fixed ad hoc single magnetic anisotropic axis. The interplay between MD and spin dynamics is small because of the low temperature regime explored, which prevents strong magnetoelastic coupling. Our MMD simulations open new possibilities for probing the complex dynamics of highly magnetostrictive materials at different temperatures.
*Correspondence author: pascal.thibaudeau@cea.fr

${ }^{1}$ B. Cantor, ed., Novel Nanocrystalline Alloys and Magnetic Nanomaterials, Series in Materials Science and Engineering (Institute of Physics, Bristol, PA, 2005).

${ }^{2}$ S. S. P. Parkin, M. Hayashi, and L. Thomas, Science 320, 190 (2008).

${ }^{3}$ S. L. Dudarev, R. Bullough, and P. M. Derlet, Phys. Rev. Lett. 100, 135503 (2008).

${ }^{4}$ M. Lines, Phys. Rep. 55, 133 (1979).

${ }^{5}$ R. F. Sabiryanov and S. S. Jaswal, Phys. Rev. Lett. 83, 2062 (1999).

${ }^{6}$ E. Rossi, O. G. Heinonen, and A. H. MacDonald, Phys. Rev. B 72, 174412 (2005).

${ }^{7}$ A. Aharoni, Introduction to the Theory of Ferromagnetism (Oxford Science, New York, 1996).

${ }^{8}$ L. A. Turski, Phys. Rev. A 30, 2779 (1984).

${ }^{9}$ V. P. Antropov, M. I. Katsnelson, M. van Schilfgaarde, and B. N. Harmon, Phys. Rev. Lett. 75, 729 (1995).

${ }^{10}$ V. P. Antropov, M. I. Katsnelson, B. N. Harmon, M. van Schilfgaarde, and D. Kusnezov, Phys. Rev. B 54, 1019 (1996).

${ }^{11}$ S. Akbar, Y. Kakehashi, and N. Kimura, J. Appl. Phys. 81, 3862 (1997).

${ }^{12}$ D. Steiauf and M. Fähnle, Phys. Rev. B 72, 064450 (2005).

${ }^{13}$ G. J. Ackland, J. Nucl. Mater. 351, 20 (2006).

${ }^{14}$ S. L. Dudarev and P. M. Derlet, J. Phys.: Condens. Matter 17, 7097 (2005).

${ }^{15}$ E. Carpene, E. Mancini, C. Dallera, M. Brenna, E. Puppin, and S. De Silvestri, Phys. Rev. B 78, 174422 (2008).

${ }^{16}$ N. Kazantseva, U. Nowak, R. W. Chantrell, J. Hohlfeld, and A. Rebei, Eur. Phys. Lett. 81, 27004 (2008).

${ }^{17}$ I. P. Omelyan, I. M. Mryglod, and R. Folk, Phys. Rev. E 65, 056706 (2002).

${ }^{18}$ I. P. Omelyan, I. M. Mryglod, and R. Folk, Comput. Phys. Comm. 146, 188 (2002).

${ }^{19}$ I. P. Omelyan, I. M. Mryglod, and R. Folk, Comput. Phys. Comm. 151, 272 (2003).

${ }^{20}$ I. P. Omelyan, I. M. Mryglod, and R. Folk, Eur. Phys. Lett. 52, 603 (2000).

${ }^{21}$ I. P. Omelyan, W. Fenz, I. M. Mryglod, and R. Folk, Phys. Rev. Lett. 94, 045701 (2005).

${ }^{22}$ P. W. Ma, C. H. Woo, and S. L. Dudarev, Philos. Mag. 89, 2921 (2009).
${ }^{23}$ P. W. Ma and C. H. Woo, Phys. Rev. E 79, 046703 (2009).

${ }^{24}$ P. Bruno, J. Phys. F: Met. Phys. 18, 1291 (1988).

${ }^{25}$ E. Du Tremolet de Lacheisserie, Magnetostriction, Theory and Applications of Magnetoelasticity (CRC Press, Boca Raton, FL, 1993).

${ }^{26}$ P. W. Ma, C. H. Woo, and S. L. Dudarev, Phys. Rev. B 78, 024434 (2008).

${ }^{27}$ H. J. Zeiger and G. W. Pratt, Magnetic Interactions in Solids (Oxford University Press, New York, 1973).

${ }^{28}$ S. V. Halilov, H. Eschrig, A. Y. Perlov, and P. M. Oppeneer, Phys. Rev. B 58, 293 (1998).

${ }^{29}$ I. P. Omelyan, I. M. Mryglod, and R. Folk, Phys. Rev. Lett. 86, 898 (2001).

${ }^{30}$ R. H. Victora and J. M. MacLaren, Phys. Rev. B 47, 11583 (1993).

${ }^{31}$ P. Tolédano, G. Krexner, M. Prem, H. P. Weber, and V. P. Dmitriev, Phys. Rev. B 64, 144104 (2001).

${ }^{32}$ T. Moriya, Phys. Rev. 120, 91 (1960).

${ }^{33}$ H. J. F. Jansen, Phys. Rev. B 38, 8022 (1988).

${ }^{34}$ M. Heide, G. Bihlmayer, and S. Blugel, Physica B 404, 2678 (2009).

${ }^{35}$ M. Finnis and J. Sinclair, Philos. Mag. A 50, 45 (1984).

${ }^{36} \mathrm{P}$. Thibaudeau and J. D. Gale, An embedded-atom method model for liquid $\mathrm{Co}, \mathrm{Nb}, \mathrm{Zr}$ and supercooled binary alloys (2008); arXiv:0809.0198v1 [cond-mat.mtrl-sci] (unpublished).

${ }^{37}$ C. Goyhenex and H. Bulou, Phys. Rev. B 63, 235404 (2001).

${ }^{38}$ T. Kaneyoshi, Amorphous Magnetism (CRC Press, Boca Raton, FL, 1984).

${ }^{39}$ D. S. G. Bauer, P. Mavropoulos, S. Louinis, and S. Blugel, J. Phys.: Condens. Matter 23, 394204 (2011).

${ }^{40}$ M. Pajda, J. Kudrnovsky, I. Turek, V. Drchal, and P. Bruno, Phys. Rev. B 64, 174402 (2001).

${ }^{41}$ O. Sipr, S. Bornemann, J. Minar, S. Polesya, V. Popescu, A. Simunek, and H. Ebert, J. Phys.: Condens. Matter 19, 096203 (2007).

${ }^{42}$ P. Mavropoulos, S. Lounis, and S. Blugel, Phys. Status Solidi B 5, 1187 (2010).

${ }^{43}$ R. Skomski, Simple Models of Magnetism (Oxford University Press, New York, 2008).

${ }^{44}$ J. B. Staunton, L. Szunyogh, A. Buruzs, B. L. Gyorffy, S. Ostanin, and L. Udvardi, Phys. Rev. B 74, 144411 (2006).

${ }^{45}$ G. Autes, C. Barreteau, D. Spanjaard, and M.-C. Desjonqures, J. Phys.: Condens. Matter 18, 6785 (2006). 
${ }^{46}$ K. H. Yang and J. O. Hirschfelder, Phys. Rev. A 22, 1814 (1980).

${ }^{47}$ J. Oteo and J. Ros, J. Phys. A: Math. Gen. 24, 5751 (1991).

${ }^{48}$ D. Frenkel and B. Smit, Understanding Molecular Simulation; From Algorithms to Applications (Academic Press, New York, 1996).

${ }^{49}$ M. Krech, A. Bunker, and D. Landau, Computer Phys. Comm. 111, 1 (1998).

${ }^{50}$ R. MacLachlan and G. Quispel, J. Phys. A: Math. Gen. 39, 5251 (2006).

${ }^{51}$ A. Dullweber, B. Leimkuhler, and R. McLachlan, J. Chem. Phys. 107, 5840 (1997).

${ }^{52}$ B. Koopmans, G. Malinowski, F. Dalla Longa, D. Steiauf, M. Fähnle, T. Roth, M. Cinchetti, and M. Aeschlimann, Nat. Mater. 9, 259 (2010).

${ }^{53}$ I. P. Omelyan, I. M. Mryglod, and R. Folk, Condens. Matter Phys. 3, 497 (2000).

${ }^{54}$ I. P. Omelyan, I. M. Mryglod, and R. Folk, Phys. Rev. E 64, 016105 (2001).

${ }^{55}$ L. E. Reichl, A Modern Course in Statistical Physics (Edward Arnold, London, 1980).

${ }^{56}$ P. W. Ma, W. C. Liu, C. H. Woo, and S. L. Dudarev, J. Appl. Phys. 101, 073908 (2007).

${ }^{57}$ H. J. C. Berendsen, J. P. M. Postma, W. F. van Gunsteren, A. DiNola, and J. R. Haak, J. Chem. Phys. 81, 3684 (1984).

${ }^{58}$ H. C. Andersen, J. Chem. Phys. 72, 2384 (1980).

${ }^{59}$ S. Nosé, J. Chem. Phys. 81, 511 (1984).

${ }^{60}$ W. G. Hoover, Phys. Rev. A 31, 1695 (1985).

${ }^{61}$ D. Kusnesoz and A. Bulgac, Ann. Phys. 214, 180 (1992).
${ }^{62}$ T. Arponen and B. Leimkuhler, BIT Num. Math. 44, 403 (2004).

${ }^{63}$ P. Thibaudeau and D. Beaujouan, Physica A 391, 1963 (2012).

${ }^{64}$ W. F. Brown, Phys. Rev. 130, 1677 (1963).

${ }^{65}$ P. W. Ma, S. L. Dudarev, A. A. Semenov, and C. H. Woo, Phys. Rev. E 82, 031111 (2010).

${ }^{66}$ J. L. García-Palacios and F. J. Lázaro, Phys. Rev. B 58, 14937 (1998).

${ }^{67}$ W. B. Nurdin and K. D. Schotte, Phys. Rev. E 61, 3579 (2000).

${ }^{68}$ P. Giannozzi, S. Baroni, N. Bonini, M. Calandra, R. Car, C. Cavazzoni, D. Ceresoli, G. L. Chiarotti, M. Cococcioni, I. Dabo, A. Dal Corso, S. de Gironcoli, S. Fabris, G. Fratesi, R. Gebauer, U. Gerstmann, C. Gougoussis, A. Kokalj, M. Lazzeri, L. MartinSamos, N. Marzari, F. Mauri, R. Mazzarello, S. Paolini, A. Pasquarello, L. Paulatto, C. Sbraccia, S. Scandolo, G. Sclauzero, A. P. Seitsonen, A. Smogunov, P. Umari, and R. M. Wentzcovitch, J. Phys.: Condens. Matter 21, 395502 (2009).

${ }^{69}$ P. Gambardella, A. Dallmeyer, K. Maiti, M. Malagoli, W. Eberhardt, K. Kern, and C. Carbone, Nature 416, 301 (2002).

${ }^{70}$ E. C. Stoner and E. P. Wolhlfarth, Phys. Trans. R. Soc. A 240, 599 (1948).

${ }^{71}$ D. Hinzke and U. Nowak, Phys. Rev. B 61, 6734 (2000).

${ }^{72}$ S. Baud, G. Bihlmayer, S. Blugel, and C. Ramseyera, Surf. Sci. 600, 4301 (2006).

${ }^{73}$ Y. Yuzhelevski, M. Yuzhelevski, and G. Jung, Rev. Sci. Inst. 71, 1681 (2000).

${ }^{74}$ U. Nowak, O. N. Mryasov, R. Wieser, K. Guslienko, and R. W. Chantrell, Phys. Rev. B 72, 172410 (2005).

${ }^{75}$ D. Beaujouan and P. Thibaudeau, J. Appl. Phys. 111, $07 \mathrm{D} 126$ (2012). 